ORI GI NAL

\title{
Niveles de progesterona durante el ciclo normal y silencioso en bovinos en el trópico colombiano
}

\section{Progesterone levels during the normal and silent cycle in cattle in the Colombian tropics}

Henry Grajales L, ${ }^{1 *}$ Ph.D, Aureliano Hernández V, ${ }^{1}$ Ph.D, Esperanza Prieto, ${ }^{2}$ M.Sc.

\begin{abstract}
${ }^{1}$ Universidad Nacional de Colombia, Facultad de Medicina Veterinaria y de Zootecnia Departamento de Ciencias para la Producción Animal, Departamento de Ciencias para la Producción Animal, Grupo de investigación en Fisiología de la Reproducción. Bogotá, Colombia. ${ }^{2}$ Corporación Colombiana de Investigación Agropecuaria - CORPOICA. Colombia. *Correspondencia: hagrajalesI@unal.edu.co
\end{abstract}

Recibido: Marzo 6 de 2009; Aceptado Diciembre 17 de 2009.

\section{RESUMEN}

Objetivo. Determinar la secuencia de cambios en los niveles de progesterona (NP) durante el Ciclo Normal (CN) y Ciclo Silencioso (CS). Materiales y métodos. 94 Hembras de los grupos raciales Holstein x Cebú (HC), Simmental x Cebú (SC), Romosinuano (R) y Cebú (C) se estudiaron desde la pubertad y durante los tres primeros ciclos estrales. Se tomaron muestras de sangre para determinar las concentraciones de progesterona mediante Radioinmunoanálisis, se determinaron 184 perfiles de progesterona para CN y 153 para CS. Para el análisis de la información se utilizó el programa estadístico SAS. Resultados. EI grupo R presenta un patrón de variaciones de los NP a través de las fases del CN que tiene diferencias significativas con el comportamiento de los NP en las fases del CN de los grupos SC, HC y C. Los niveles de progesterona en cada fase del CS coinciden con una fase luteal con bajos niveles de progesterona. Solo se encontraron diferencias significativas en la fase luteal tardía al comparar el CN con el CS, sin embargo se presentan diferencias numéricas en todas las fases entre estos dos tipos de ciclos. Conclusiones. Las concentraciones de progesterona tienen un marcado efecto sobre la presentación del CS, no obstante es difícil definir una concentración de progesterona que caracterice cada evento (CN o CS), sin embargo, es evidente que la progesterona influye sobre las probabilidades para que se de una respuesta reproductiva favorable o desfavorable.

Palabras clave: Ciclo estral, ciclo silencioso, genotipo, progesterona, trópico. 


\section{ABSTRACT}

Objective. Determine the sequence of changes in the levels of progesterone (NP) during the normal cycle (NC) and Quiet Cycle (CS). Materials and methods. 94 Females of the breed Holstein $x$ Cebu (HC), Simmental x Cebu (SC), Romosinuano (R) and Cebu (C) were studied from puberty and during the first three estrous cycles. Blood samples were taken to determine concentrations of progesterone by radioimmunoassay, 184 progesterone profiles were determined for $\mathrm{CN}$ and 153 for CS. For the analysis of the information the statistical program SAS was used. Results. The R group presented a pattern of variations of the NP through the phases of the $\mathrm{CN}$ that has significant differences with the behavior of NP in the phases of CN groups SC, HC and C. Progesterone levels in each phase of the CS are consistent with a low luteal phase progesterone levels. Only significant differences were found in the late luteal phase when comparing CN with CS, however, numerical differences arise at all stages between these two types of cycles.. Conclusions. Progesterone concentrations have a marked effect on the presentation of CS, however, is difficult to define a concentration of progesterone that characterize each event (CN or CS), however, it is clear that progesterone affects the chances to be a favorable or unfavorable reproductive response.

Key words: genotype, oestrus cycle, progesterone, quiet cycle, tropic.

\section{NTRODUCCI ÓN}

El celo silencioso es una condición en la cual los órganos genitales sufren cambios cíclicos normales, pero los signos de comportamiento del estro no son manifestados $(1,2)$. Se ha reportado que la primera y la segunda ovulación posparto no son acompañadas por los signos del estro, y son consideradas como celos silenciosos "verdaderos", así mismo se plantea que es poco probable que más "celos silenciosos verdaderos" ocurran después del segundo ciclo $(3,4)$. Sin embargo, varios autores reportan la incidencia de celos silenciosos posteriores al periodo posparto, Arthur et al (3), reportaron una incidencia de $10.6 \%$ de celos silenciosos, incluso cuando las vacas eran examinadas cuatro veces en 24 horas para determinar la presencia del celo $(3,5)$. I gualmente reportaron que el $23.7 \%$ de 3076 ovulaciones, provenían de celos silenciosos (3). Kidder et al (6) y Dawuda et al (7), reportaron que la presencia de celos silenciosos era del $44 \%$ durante los primeros 60 días después del parto y del $11 \%$ entre los 61 a 308 días posparto.

Se ha reconocido que la expresión de los signos del celo en bovinos es afectada principalmente por factores ambientales, interacciones sociales, estrés, factores genéticos y las concentraciones de progesterona (8).

Los factores ambientales tales como la duración del día (fotoperiodo), periodo de lluvias, fuertes vientos y altas temperaturas, influencian la intensidad del estro, la receptividad sexual y la eficiencia reproductiva en los bovinos (9).

Las interacciones sociales dentro de un hato, desempeña un papel importante en la exhibición del comportamiento estral; el estatus jerárquico de una vaca parece ejercer una fuerte influencia en la expresión del estro Orihuela (8) afirma que las hembras dominantes dentro de un hato inhiben la actividad de monta de las hembras subordinadas. Galina et al (10) indicaron que el número de vacas en estro afecta la frecuencia de monta y la duración del estro, reportando que el número promedio de montas por vaca y la duración de los signos del estro incrementa cuando dos o más animales son sexualmente receptivos al mismo tiempo.

El estrés impacta negativamente la función ovárica, ya que inhibe la secreción de la hormona liberadora de las gonadotropinas 
( $\mathrm{GnRH}$ ) a nivel hipotalámico, en consecuencia suprime la liberación de la hormona luteinizante (LH) a nivel hipofisiario, lo que a su vez afecta las concentraciones de estradiol y progesterona, alterando así la intensidad del comportamiento estral (11).

Existen diferencias entre especies y razas con respecto a la duración y la intensidad del comportamiento estral. La duración del estro en ganado Bos indicus es significativamente menor (11horas) que en ganado Bos taurus (18 horas) (12). Diferencias en la intensidad del estro entre ganado Bos taurus y Bos indicus ha sido reportada, en general el comportamiento estral es menos intenso en el ganado tropical (8). El ganado Bos indicus por lo general tiene un temperamento muy particular que dificulta la detección del celo, celos silenciosos han sido reportados, incluso después de una detección regular de calores $(10,13)$.

Existe una correlación significativa entre las concentraciones de estradiol y la expresión del comportamiento de celo, de igual manera la progesterona juega un papel crítico en la expresión de este comportamiento. La progesterona puede inhibir o favorecer la respuesta en comportamiento provocada por el estradiol dependiendo del tiempo de exposición, así como altas concentraciones de progesterona durante la fase luteal incrementa en número de receptores de estradiol en el hipotálamo medio-basal y por lo tanto incrementa la sensibilidad al estradiol. Por lo tanto el "priming" de progesterona es necesario para que se de la expresión normal del comportamiento de celo (14), en consecuencia, la baja intensidad del estro presente en algunos animales puede estar directamente relacionada con una insuficiente exposición a la progesterona antes del esto (10).

Existe poca información acerca del comportamiento de las concentraciones de progesterona y su relación con la expresión del comportamiento de celo es escasa en condiciones de trópico cálido-húmedo en ganado de doble propósito, criollo y cebú.

El objetivo de esta investigación fue determinar la secuencia de cambios en los niveles de progesterona sérica (NP) con ganado bovino del tropico colombiano.

\section{MATERI ALES Y MÉTODOS}

Sitio de estudio. El trabajo experimental se realizó en el centro de investigaciones Turipaná de la Corporación Colombiana para la Investigación Agropecuaria (CORPOICA), ubicado en el nor-occidente colombiano (trópico cálido-húmedo) a 20 msnm, con una temperatura promedio de $27.5^{\circ} \mathrm{C}, 1200 \mathrm{~mm} /$ año de precipitación y una humedad relativa promedio del $83 \%$.Geográficamente se encuentra a $8^{\circ} 5^{\prime}$ de latitud norte y $75^{\circ} 49^{\prime}$ de longitud occidente, con respecto al meridiano de Greenwich.

Animales. Se escogieron al azar 94 novillas destetadas ( 8 a 14 meses de edad), así: 14 $F_{1}$ Simmental $x$ Cebú $(\mathrm{SC}) ; 28 \mathrm{~F}_{1}$ Holstein $\mathrm{x}$ Cebú (HC); 40 Romosinuano (R) y 12 Cebú (C). Los animales se estudiaron a partir de la determinación de la pubertad (primer calor observado) y durante los tres (3) primeros ciclos estrales completos consecutivos observados por animal, realizando seguimiento individual de los animales dos veces al día durante una hora, de 6 a 7 a.m. y de 5 a 6 p.m., para registrar la presencia de calores (una hembra se consideró en celo cuando se dejó montar dos o más veces por otras hembras o por el macho marcador o cuando montó a otras hembras); todos los animales fueron pesados cada quince días y al momento de presentar los celos.

Manejo de los animales. Los animales fueron mantenidos en praderas mixtas compuestas por, Dichantium aristatum (Angleton) y, Brachiaria mutica (Pará), con acceso a sal mineralizada ( $8 \% \mathrm{P})$ y agua a voluntad; se realizaron controles periódicos para determinar la composición y disponibilidad de forraje, para mantener el cubrimiento de las necesidades nutricionales de los animales y procurar durante todo el periodo de seguimiento un comportamiento fisiológico normal.

Determinación de perfiles de progesterona. Se determinaron un total de 337 perfiles de progesterona en las hembras evaluadas, 
correspondiendo 184 a CN y 153 a CS.

Obtención de muestras y radioinmunoanálisis. Después de la aparición del primer calor observado se colectaron muestras de sangre venosa, mediante la técnica de venopunción de la yugular así: día $0=$ FF (día de presentación del calor) y los días $5=$ FLtp, $10=$ FLm, $15=$ FLtr y $20=$ FF de cada ciclo estral y cada cinco días hasta la aparición del siguiente calor, durante los tres primeros ciclos estrales completos consecutivos observados por animal. Las muestras de sangre, se centrifugaron a 2500 rpm por 15 minutos para separar el suero de los elementos formes; el suero se almacenó en viales y se mantuvo en congelación $\left(-20^{\circ} \mathrm{C}\right)$ hasta la cuantificación de los niveles de progesterona por la técnica del radioinmunoanálisis en fase sólida (RIA) $(15,16)$, para lo cual se utilizaron kits CoatA-Count Progesterone de la Diagnostics Products Corporation (DPC), el cual presenta una sensibilidad de $0.02 \mathrm{ng} / \mathrm{ml}$ y una reactividad cruzada muy baja con otros componentes presentes en la muestra. Las muestras fueron corridas en secuencias diferentes, obteniéndose un coeficiente de variación intra-ensayo de $8.79 \%$ e interensayo de $10.38 \%$.

Criterios para manejo de variables. El ciclo normal (CN) se definió como el comportamiento estral con manifestación de calor observado, que estuvo respaldado por una función luteal basada en el mantenimiento de los niveles de progesterona por encima de $2 \mathrm{ng} /$ $\mathrm{ml}$ presente por un periodo de tiempo determinado o de manera cíclica $(15,17,18)$; el ciclo silencioso (CS) se definió como el comportamiento estral sin manifestación de calor observado, que estuvo respaldado por una función luteal basada en el mantenimiento de los niveles de progesterona por encima de $2 \mathrm{ng} / \mathrm{ml}$ presente en una o dos muestras consecutivas (15, 17-19).

Análisis estadístico. Utilizando estadística descriptiva se observó y analizó el comportamiento de las variables descritas dentro y entre los grupos raciales, usando para el análisis el procedimiento GLM del programa de análisis estadístico SAS (20).Para comparar el comportamientos de los NP en las fases del ciclo estral, entre los grupos raciales evaluados, se efectuó un análisis de varianza para medidas repetidas en el tiempo, determinando el paralelismo de las curvas de progesterona a través de los tiempos de muestreo (20).

\section{RESULTADOS}

Niveles de progesterona en ciclo normal (CN). Al comparar el comportamiento de los NP en las fases del CN (Tabla1), las variaciones entre promedios en los tiempos puntuales evaluados (fases: días $0,5,10,15$ y 20) no presentaron diferencias significativas $(p>0.05)$ entre los grupos SC y HC, indicando que los comportamientos de los NP para estos dos grupos son similares. Los análisis comparativos entre los otros grupos permitieron determinar que se presentan diferencias significativas en la FLtp $(p<0.01)$ y la FLm $(p<0.5)$ entre el grupo SC (2.716 y $5.140 \mathrm{ng} / \mathrm{ml}$ ) y el grupo $\mathrm{R}$ ( 1.916 y $5.908 \mathrm{ng} / \mathrm{ml}$ ), así como también, en la FLtr $(p<0.01)$ entre el grupo SC $(6.701 \mathrm{ng} /$ $\mathrm{ml}$ ) y el grupo C (4.959 $\mathrm{ng} / \mathrm{ml})$. El grupo HC tuvo diferencias significativas con el grupo $\mathrm{R}$ en la FLtp $(p<0.05)(2.467$ vs $1.916 \mathrm{ng} / \mathrm{ml})$ y en la FLtr $(p<0.05)(5.750$ vs $6.624 \mathrm{ng} / \mathrm{ml})$ y con el grupo $C$ en la FLm $(p<0.01)$ (5.512 vs $4.348 \mathrm{ng} / \mathrm{ml})$ y en la FLtr $(p<0.01) \quad(5.750$ vs $4.959 \mathrm{ng} / \mathrm{ml}$ ). Por su parte, el grupo R presentó diferencias significativas con el grupo $C$, a nivel de la FLtp, en donde su NP fue de $2.345 \mathrm{ng} / \mathrm{ml}$ $(p<0.05)$, en la FLm fue $4.348 \mathrm{ng} / \mathrm{ml}(p<0.01)$ y en la FLtr fue $4.959(p<0.01)$.

El análisis de varianza para medidas repetidas, usando la prueba de paralelismo, permitió demostrar que se presentan diferencias entre razas en el comportamiento de las curvas de

Tabla 1. Niveles de progesterona (NP) (ng/ml) para las fases del $\mathrm{CN}$ en bovinos en el trópico cálido-húmedo colombiano.

\begin{tabular}{cccccc}
\hline \multirow{2}{*}{$\begin{array}{c}\text { Grupo } \\
\text { racial }\end{array}$} & FF & FLtp & FLm & FLtr & FF \\
\hline SC(n=39) & $0.374 \mathrm{a}$ & $2.716 \mathrm{a}$ & $5.140 \mathrm{a}$ & $6.701 \mathrm{a}$ & $0.641 \mathrm{a}$ \\
& \pm 0.31 & \pm 1.45 & \pm 2.04 & \pm 2.77 & \pm 0.82 \\
& $0.331 \mathrm{a}$ & $2.467 \mathrm{a}$ & $5.512 \mathrm{a}$ & $5.750 \mathrm{a}$ & $0.560 \mathrm{a}$ \\
$\mathbf{H C}(\mathbf{n = 6 0})$ & \pm 0.36 & \pm 1.46 & \pm 2.12 & \pm 1.94 & \pm 0.78 \\
& $0.221 \mathrm{a}$ & $1.916 \mathrm{~b}^{*}$ & $5.908 \mathrm{ab}^{*}$ & $6.624 \mathrm{ab}^{*}$ & $0.381 \mathrm{a}$ \\
$\mathbf{R}(\mathbf{n = 5 4 )}$ & \pm 0.20 & \pm 0.96 & \pm 2.01 & \pm 2.67 & \pm 0.53 \\
& $0.165 \mathrm{a}$ & $2.345 \mathrm{ac}^{* *}$ & $4.34 \mathrm{ac}^{* *}$ & $4.959 \mathrm{c}^{* *}$ & $0.484 \mathrm{a}$ \\
$\mathbf{C}(\mathbf{n = 3 1 )}$ & \pm 0.11 & \pm 1.05 & \pm 0.68 & \pm 1.43 & \pm 0.94 \\
\hline
\end{tabular}

$\mathrm{CN}=$ Ciclo normal; $\mathrm{SC}=$ Simmental $\times$ Cebú; $\mathrm{HC}=$ Holstein $x$ Cebú; $\mathrm{R}=$ Romosinuano; $\mathrm{C}=$ Cebú. $\mathrm{FF}=$ Fase Folicular (día 0); FLtp=Fase luteal temprana (día 5); FLm=Fase luteal media (día 10); FLtr=Fase luteal tardía (día $15) .^{*}=p<0.05(a=0.5 \%) ; * *=p<0.001 \quad(a=0.01 \%)$. 
progesterona, estando la respuesta afectada por la interacción del factor raza (grupo racial) - tiempo de muestreo (fase del ciclo estral) (Figura 1).

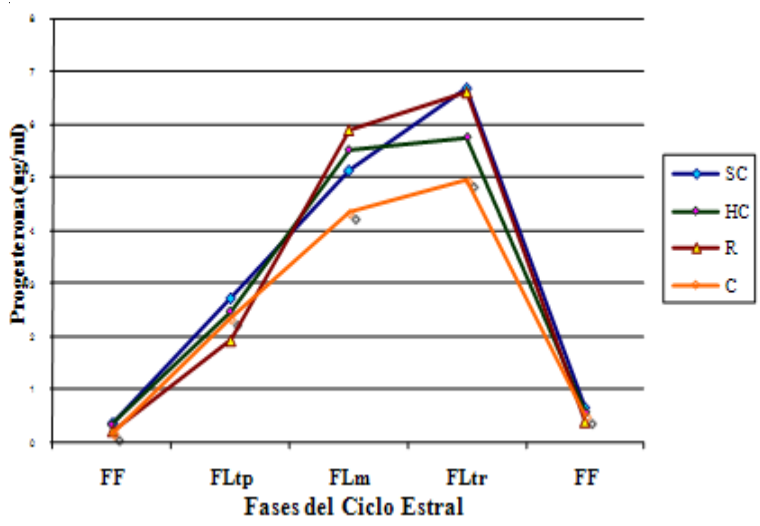

Figura 1. Niveles de progesterona en las fases del CN para bovinos en el trópico cálido húmedo colombiano. ( $\mathrm{CN}=$ Ciclo Normal; $\mathrm{SC}=$ Simmental $\times$ Cebú; $\mathrm{HC}=$ Holstein $x$ Cebú; $\mathrm{R}=$ Romosinuano; $\mathrm{C}=$ Cebú; $\mathrm{FF}=$ Fase Folicular (día 0); FLtp=Fase luteal temprana (día 5); FLm=Fase luteal media (día 10); FLtr=Fase luteal tardía (día 15).

El grupo R presenta un patrón de variaciones de los NP a través de las fases del CN que tiene diferencias significativas con el patrón de comportamiento de los NP en las fases del CN que mostraron los grupos SC, HC y C (Tabla 1 ). En términos generales se puede observar como el grupo R partiendo de NP bajos en la FLtp, logra el mayor nivel de variación de la actividad luteal en las FLm y FLtr, mostrando un cambio en la tendencia que describe la curva que es diferente al de los otros grupos (Figura 2).

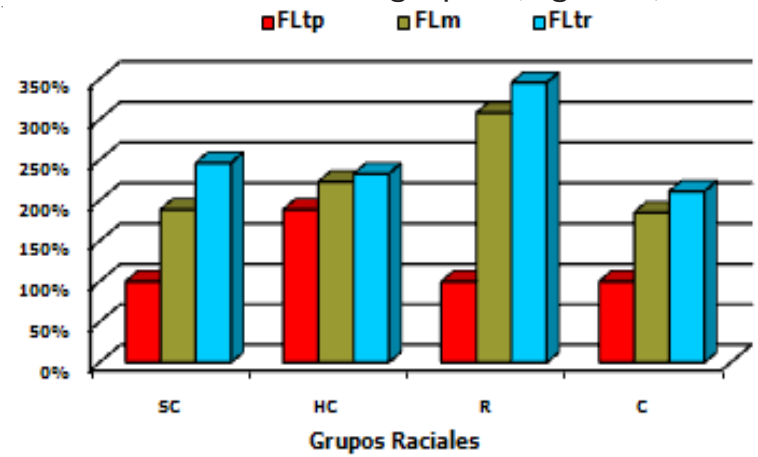

Figura 2. Porcentaje de variación de la actividad luteal a través de las fases del CN $(\mathrm{CN}=$ Ciclo Normal; $\mathrm{SC}=$ Simmental $\mathrm{x}$ Cebú; $\quad H C=$ Holstein $x$ Cebú; $\mathrm{R}=$ Romosinuano; $\mathrm{C}=$ Cebú; FLtp=Fase luteal temprana; $\mathrm{FLm}=$ Fase luteal media; FLtr=Fase luteal tardía).
Mientras el grupo R presenta una variación proporcional de magnitud importante, de la FLtp a la FLm de $308.35 \%$ y a la FLtr de $345.72 \%$, los otros grupos apenas alcanzan una variación de $223.43 \%$ y $233.08 \%$ para el HC; $189.25 \%$ y $246.72 \%$ para el SC; y, $185.4 \%$ y $211.47 \%$ el C, respectivamente para las mismas fases (Figura 2).

Niveles de progesterona en ciclo silencioso (CS). Los niveles de progesterona determinados en cada una de las fases del CS (Tabla 2) se caracterizan por ser bajos durante toda la fase luteal. Al comparar el comportamiento de los NP en las fases del CS, las variaciones entre promedios en los tiempos puntuales evaluados (fases: días 0 , $5,10,15$ y 20) no presentaron diferencias significativas $(p>0.05)$ entre el grupo SC con los grupos HC y $C$, indicando que los comportamientos de los NP para estos grupos es muy similar (Figura 3), excepto en la FLm, en donde se presentan diferencias significativas $(p<0.05)$ entre el grupo $\mathrm{HC}(5.154 \mathrm{ng} / \mathrm{ml})$ y el C ( $4.322 \mathrm{ng} / \mathrm{ml})$.

Tabla 2. Niveles de progesterona (NP) $(\mathrm{ng} / \mathrm{ml})$ para las fases del CS en bovinos en el trópico cálido-húmedo colombiano.

\begin{tabular}{cccccc}
\hline $\begin{array}{c}\text { Grupo } \\
\text { racial }\end{array}$ & FF & FLtp & FLm & FLtr & FF \\
\hline \multirow{2}{*}{$\mathbf{S C}(\mathbf{n = 2 4 )})$} & $0.279 a$ & $2.473 a$ & $4.662 a$ & $3.768 a$ & $0.202 a$ \\
& \pm 0.11 & \pm 1.13 & \pm 2.28 & \pm 1.32 & \pm 0.07 \\
& $0.254 a$ & $2.466 a$ & $5.154 a$ & $3.502 a$ & $0.272 a$ \\
HC(n=44) & \pm 0.12 & \pm 1.17 & \pm 2.32 & \pm 1.54 & \pm 0.11 \\
& $0.202 a$ & $2.944 a$ & $5.517 a b^{*}$ & $4.512 a b^{*}$ & $0.247 a$ \\
$\mathbf{R}(\mathbf{n = 4 3 )}$ & \pm 0.09 & \pm 1.37 & \pm 2.04 & \pm 2.18 & \pm 0.04 \\
& & & $4.322 a c$ & $3.905 a c^{* *}$ & $0.210 a$ \\
C(n=42) & $0.239 a$ & $2.197 a b^{*}$ & $(*)\left(^{* *}\right)$ & \pm 1.52 & \pm 0.06 \\
& \pm 0.07 & \pm 0.05 & \pm 1.09 & & \\
\hline
\end{tabular}

$\mathrm{CS}=$ Ciclo silencioso; $\mathrm{SC}=$ Simmental $\times$ Cebú; $\mathrm{HC}=$ Holstein $\mathrm{x}$ Cebú; $\mathrm{R}=$ Romosinuano; $\mathrm{C}=$ Cebú. $\mathrm{FF}=$ Fase Folicular (día 0); FLtp=Fase luteal temprana (día 5); FLm=Fase luteal media (día 10); FLtr=Fase luteal tardía (día 15).* $=p<0.05 \quad(a=0.5 \%)$; $* *=p<0.001 \quad(a=0.01 \%)$.

Los demás análisis comparativos entre los otros grupos permitieron determinar que se presentan diferencias significativas en la FLm $(p<0.05)$ entre el grupo SC (4.662 ng/ml) y el grupo R $(5.517 \mathrm{ng} / \mathrm{ml})$; el grupo HC tuvo diferencias significativas con el grupo R en la FLtr $(p<0.05)$ ( 3.502 vs $4.512 \mathrm{ng} / \mathrm{ml}$ ) y con el grupo $C$ en la FLm ( $p<0.05)$ (5.154 vs $4.322 \mathrm{ng} / \mathrm{ml})$. Por su parte, el grupo $\mathrm{R}$ presentó diferencias significativas con el grupo C, en la FLtp, en 


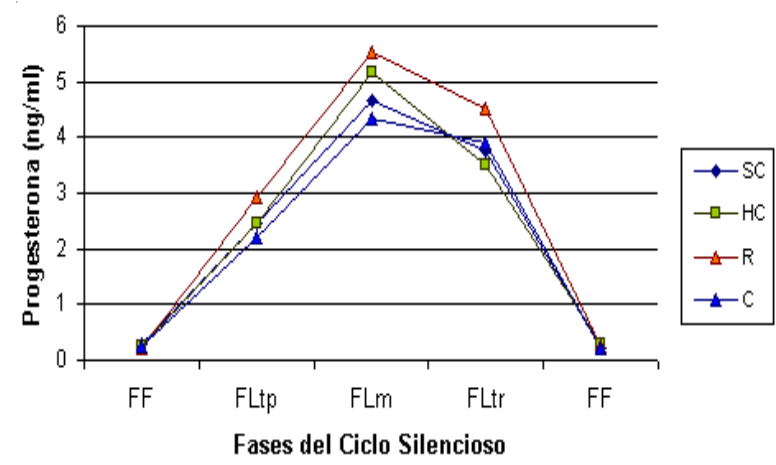

Figura 3. Niveles de progesterona en las fases del CS para bovinos en el trópico cálido húmedo colombiano. ( $\mathrm{CS}=$ Calor Silencioso; $\mathrm{SC}=$ Simmental $\times$ Cebú; $\mathrm{HC}=$ Holstein $\times$ Cebú; $\mathrm{R}=$ Romosinuano; $\mathrm{C}=$ Cebú; $\mathrm{FF}=$ Fase Folicular (día 0); FLtp=Fase luteal temprana (día 5); FLm=Fase luteal media (día 10); FLtr=Fase luteal tardía (día 15).

donde su NP fue de 2.944 vs $2.197 \mathrm{ng} / \mathrm{ml}$ $(p<0.05)$ respectivamente, en la FLm con 5.517 vs $4.322 \mathrm{ng} / \mathrm{ml}(\mathrm{p}<0.01)$ y en la FLtr con 4.512 vs $3.905 \mathrm{ng} / \mathrm{ml}(p<0.01)$.

El grupo C tuvo la mayor proporción de CS con respecto al total de ciclos determinados para la población en estudio con $45.6 \%$ en el $81.8 \%$ de las novillas y en el $78.9 \%$ de las vacas; respecto a un $31.6 \%$ para el grupo $\mathrm{R}$ en el $61.1 \%$ de las novillas y el $66.6 \%$ de las vacas; a un $21.8 \%$ para el grupo $\mathrm{HC}$ en el $42.8 \%$ de las novillas y el $28 \%$ de las vacas; $y$, un $19.8 \%$ para el grupo SC en el $76.9 \%$ de las novillas y el $45.4 \%$ de las vacas.

Comportamiento de los niveles de progesterona en los tipos de ciclos evaluados. Al hacer el análisis del comportamiento de los NP comparando entre los tipos de ciclos clasificados no se encontraron diferencias significativas ( $p>0.05$ ) en las FF, FLtp
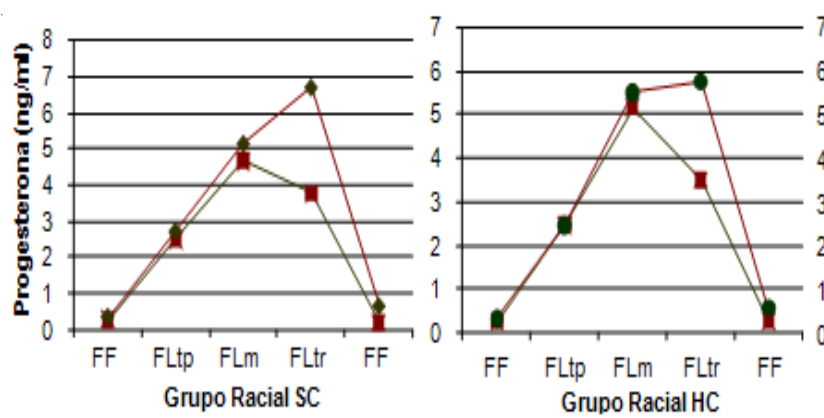

y FLm entre el CN con el CS en los que se observa que la actividad luteal inicia en un NP entre 0.192 y $0.243 \mathrm{ng} / \mathrm{ml}$ en la FF (día 0) y se ubicó en valores entre 4.914 y 5.227 en la FLm, a partir de la cual se empezaron a notar cambios en el comportamiento de la actividad luteal con diferencias significativas $(p<0.01)$ entre estos mismos tipos de ciclos, en la FLtr y FFf (día 20), encontrando en los CS un cuerpo lúteo con baja actividad luteal ( 3.922 y $0.233 \mathrm{ng} / \mathrm{ml}$ ) y en regresión, la que se produce de una manera más rápida y brusca que en los CN (6.006 y $0.516 \mathrm{ng} / \mathrm{ml}$ ) (Tabla 3) (Figura 4).

EI NP acumulado durante las fases del ciclo, representado por la suma de los valores de cada una de las fases evaluadas, puede llegar a ser un buen indicador de la variación de la actividad luteal, permitiendo hacer una clasificación exitosa del tipo de ciclicidad que se está presentando (Tabla 4).

Tabla 3. Prueba de significancia (promedios totales) para la variación de los niveles de progesterona $(\mathrm{ng} / \mathrm{ml})$ en las fases del ciclo estral en bovinos en el trópico cálidohúmedo colombiano.

\begin{tabular}{lcc}
\hline \multicolumn{1}{c}{$\begin{array}{c}\text { Fase del } \\
\text { ciclo }\end{array}$} & \multicolumn{2}{c}{ Tipo de ciclo } \\
\hline FF (día 0) & 0.243 NS & CS \\
FLtp (día 5) & 2.520 NS & 2.361 \\
Flm (día 10) & 4.914 NS & 5.227 \\
Fltr (día 15) & $3.922 * *$ & 6.009 \\
FF (día 20) & $0.233 *$ & 0.516 \\
\hline
\end{tabular}

NS=Diferencia No significativa; $*=$ Diferencia significativa $\mathrm{p}<0.05(\mathrm{a}=0.5 \%) . * *=$ Diferencia significativa $\mathrm{p}<0.001$ $(a=0.01 \%) . C N=$ Ciclo Normal; $C S=$ Ciclo Silencioso. $\mathrm{FF}=$ Fase Folicular (día 0); FLtp=Fase luteal temprana (día 5); FLm=Fase luteal media (día 10); FLtr=Fase luteal tardía (día 15).

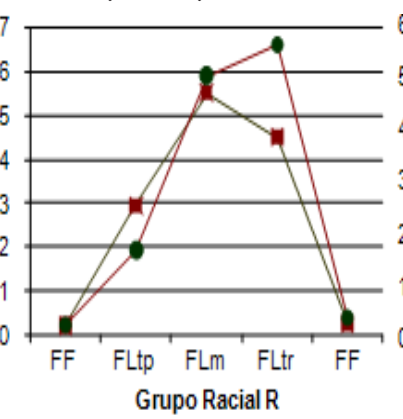

Figura 4. Niveles de progesterona en las fases del CN y CS para bovinos en el trópico cálido húmedo colombiano. ( $\mathrm{CN}=$ Ciclo Normal; $\mathrm{CS}=$ Calor Silencioso; $\mathrm{SC}=$ Simmental $\times$ Cebú; HC = Holstein x Cebú; $\mathrm{R}=$ Romosinuano; $\mathrm{C}=$ Cebú). 
Tabla 4. Niveles de progesterona acumulado (ng/ $\mathrm{ml}$ ) durante el CN y CS en bovinos en el trópico cálido-húmedo colombiano.

\begin{tabular}{ccc}
\hline $\begin{array}{c}\text { Grupo } \\
\text { Racial }\end{array}$ & \multicolumn{2}{c}{ Tipo de ciclo } \\
& $\begin{array}{c}\text { Ciclo Normal Calor Silencioso } \\
\mathrm{ng} / \mathrm{ml} \text { de P4 acumulada }\end{array}$ \\
\hline SC & 15.545 & 11.348 \\
HC & 14.620 & 11.648 \\
R & 15.050 & 13.421 \\
C & 12.301 & 10.873 \\
\hline
\end{tabular}

$\mathrm{SC}=$ Simmental $\times$ Cebú; $\mathrm{HC}=$ Holstein $\times$ Cebú; $\mathrm{R}=$ Romosinuano; $\mathrm{C}=$ Cebú.

\section{DISCUSIÓN}

En el presente estudio las concentraciones de progesterona durante el Ciclo Normal fueron bajas durante la fase folicular, incrementando a través de la fase luteal temprana, alcanzando sus valores máximos durante la fase luteal media y tardía, estos resultados concuerdan con reportes previos hechos en bovinos $(21,22)$. Sin embargo, los niveles de progesterona determinados en cada una de las fases del ciclo normal en general son mayores a los reportados en los trabajos de Prieto (15) y Cardozo (16) en novillas Holstein en la Sabana de Bogotá, pudiendo aducirse que ello obedece a una mayor actividad del cuerpo lúteo como consecuencia de la "tensión térmica" o "estrés calórico" como lo reportan Solano et al (23) y Hernández et al (24) en trabajos realizados en Cuba con novillas Holstein y en la zona tropical de México, respectivamente; al respecto, existe mucha controversia por los datos contradictorios respecto al efecto de las altas temperaturas sobre el incremento o no de los NP durante el ciclo estral y los efectos que ella puede traer sobre la tasa de concepción y la viabilidad embrionaria.

Los grupos $C$ y $R$ presentan la menor actividad luteal en la FF, comparada con lo manifestado por los grupos SC y HC, denotándose que en las FLm y FLtr el grupo R en mayor proporción que el $C$, evidencia una mayor variación de su actividad luteal para la producción de progesterona. Baez et al (25), reportan también en ganado Costeño con cuernos un patrón de variación alto en las concentraciones de progesterona a través de las fases luteal temprana y luteal media (373.71\%). El comportamiento de los NP durante las fases del CN en el grupo R, y de manera semejante en el grupo $\mathrm{C}$, se relaciona favorablemente con el desempeño reproductivo que mostraron las novillas en su tasa de concepción en primera y segunda preñez, en un estudio previo realizado con el mismo grupo animal por Grajales et al (26).

Los niveles de progesterona determinados en cada una de las fases del ciclo silencioso coinciden con una fase luteal con bajos niveles de progesterona la cual es la causa de la presencia de estros débiles o "estros silenciosos" Dhali et al (14), reportaron que el ganado Bos frontalis (animales que presentan celos silenciosos de manera natural) presenta menores concentraciones de progesterona durante la fase luteal al ser comparado con bovinos y búfalos. Asimismo en estudios realizados por Mondal et al $(22,27)$ se observó que los niveles de progesterona plasmática eran más bajos en vacas que presentaron celo silencioso comparado con vacas que exhibieron un celo normal. Se ha reportado también que la presentación de calores silenciosos está asociada a la presencia de cuerpos lúteos pequeños, que en general se reporta tienen mayor incidencia en los ganados Bos índicus que en los Bos taurus (28). En términos generales se puede observar una pobre actividad luteal durante el ciclo silencioso, si se compara a los niveles de progesterona que se presentan en un ciclo normal; en las FF, FLtp y FLm se da un incremento de la actividad luteal que disminuye anormalmente en la FLtr, indicando el inicio de la regresión funcional y estructural del cuerpo lúteo, lo que incide sobre la dinámica de crecimiento y desarrollo folicular y sobre el patrón de secreción hormonal que en últimas determina la presencia de estos celos silenciosos.

La mayor incidencia de ciclos silenciosos en el ganado cebú reportado en este trabajo puede deberse, a que este genotipo en condiciones tropicales, presenta una débil expresión de los signos del estro, una corta y variable duración del estro y un número reducido de montas por hora (29), y coincide con los reportes presentados por Orihuela (8), Galina y Orihuela (9) y Bo et al (13). 
En este trabajo se logró establecer: i. Que el patrón de variaciones de los niveles de progesterona a través de las fases del ciclo normal en el grupo R es diferente al patrón de variaciones de los niveles de progesterona en las fases del ciclo normal que mostraron los grupos SC, HC y C; ii. Que Los niveles de progesterona determinados en cada una de las fases del ciclo silencioso coinciden con una fase luteal con bajos NP la cual es la causa responsable de estros débiles frecuentemente encontrados en el ganado, denominados "estros no manifiestos"; iii. El patrón de variación de los niveles de progesterona a través de las fases del ciclo silencioso es similar en todos los grupos raciales evaluados, en términos generales se observa una fase luteal con bajos niveles de progesterona en las FF, FLtp y FLm, que disminuye anormalmente en la FLtr, indicando una anticipada regresión funcional y estructural del cuerpo lúteo; iv. Los niveles de progesterona acumulados durante las fases del ciclo, representado por la suma de los valores de cada una de las fases evaluadas, puede llegar a ser un buen indicador de la variación de la actividad luteal, permitiendo hacer una clasificación exitosa del tipo de ciclicidad que se está presentando.

Asimismo se concluye que las concentraciones de progesterona tienen un marcado efecto sobre la presentación del ciclo silencioso (CS). No obstante, es sumamente difícil definir una concentración de progesterona que caracterice cada evento. Si bien es cierto que la concentración de progesterona por sí sola no es determinante de la presentación de un calor, si es claro y evidente que ella influye sobre las probabilidades para que se de una respuesta reproductiva favorable o desfavorable.

\section{Agradecimientos}

A Colciencias por el apoyo financiero para el desarrollo del trabajo y a Corpoica por el apoyo para el desarrollo de la investigación en el Centro de Investigaciones Turipaná. Cerete, Colombia.

\section{REFERENCI AS}

1. Mwaanga ES, Janowski T. Anoestrus in dairy cows: prevalence and clinical forms. Reprod Domest Anim 2000; 35: 193-200.

2. Zdunczyk S, Piskula M, Janowski T, Baranski W, Ras M. Concentrations of isoflavones in blood plasma of dairy cows with different incidence of silent heat. Bull Vet Inst Pulawy 2005; 49: 189-191.

3. Arthur GH, Noakes DE, Parkinson TJ, England GCW. Arthur's veterinary reproduction and obstetrics. 8th ed. London, Philadelphia: Saunders Company Publisher; 2001.

4. Crowe MA. Resumption of ovarian cyclicity in post-partum beef and dairy cows. Reprod Domest Anim 2008; 43(Supl 5): 20-28.

5. Hall J L, Branton C, Stone EJ. Estrus, estrous cycles, ovulation time, time of service and fertility of dairy cattle in Louisiana. J Dairy Sci 1959; 42: 1086.
6. Kidder HE, Barrett GR Casida LE. A study of ovulation in six families of HolsteinFreisians. J Dairy Sci 1952; 35:436.

7. Dawuda PM, Eduvie LO, Esievo KAN, Molokwu ECI. Silent oestrus manifestation in Nigerian Bunaji Zebu cows. Anim Reprod Sci 1989; 21: 79-85.

8. Orihuela A. Some factors affecting the behavioural manifestation of oestrus in cattle: a review. Appl Anim Behav Sci 2000; 70: 1-16.

9. Galina CS, Orihuela A. The detection of estrus in cattle raised under tropical conditions: What we know and what we need to know. Horm Behav 2007; 52: 32-38.

10. Galina CS, Orihuela A, Rubio I. Behavioural trends affecting oestrus detection in Zebu cattle. Anim Reprod Sci 1996; 42: 465-470. 
11. Walker SL, Smith RF, J ones DN, Routly JE, Dobson H. Chronic stress, hormone profiles and estrus intensity in dairy cattle. Horm Behav 2008; 53: 493-501.

12. Verduzco A, Rubio I, Galina CS, Maquivar $M$. Average response to estrus and timing of ovulation in Bos indicus cattle synchronized alternatively with a synthetic progestagen. J Anim Vet Adv 2006; 5(11):924-929.

13. Bó GA, Baruselli PS, Martínez MF. Pattern and manipulation of folicular development in Bos indicus cattle. Anim Reprod Sci 2003; 78: 307-326.

14. Dhali A, Mishra DP, Karunakaran M, Mech A, Rajkhowa C. Influence of plasma estradiol $17-\beta$ and progesterone levels on estrous behaviour in mithun (Bos frontalis). Appl Anim Behav Sci 2006; 98: 1-10.

15. Prieto ME. Edad y peso al inicio de la pubertad y seguimiento de los tres primeros ciclos estrales en novillas Holstein [tesis de maestría]. Bogotá, Colombia: Universidad Nacional de Colombia; 1993.

16. Cardozo CJ. Relación entre factores climáticos y algunas características del ciclo estral en novillas Holstein de la Sabana de Bogotá [tesis de maestría]. Bogotá, Colombia: Universidad Nacional de Colombia; 1993.

17. Short RE, Bellows RA, Staigmiller RB, Berardinelli JG Custer EE. Physiological mechanisms controlling anestrus and infertility in postpartum beef cattle. J Anim Sci 1990; 68: 799-816.

18. Wherman ME, Kojima FN Sanchez T, Mariscal DV, Kinder JE. Incidence of precocious puberty in developing beef heifers. J Anim Sci 1996; 74:2462-2467.

19. Wilson SJ, Kirby CJ, Koenigsfeld AT, Keisler DH, Lucy MC. Effects of controlled heat stress on ovarian function of dairy cattle. 2. Heiferes. J Dairy Sci 1998; 81:2132-2138.
20. SAS/STAT: Guide for Personal Computer [programa de ordenador]. Versión 6.12. Cary (NC): SAS Institute Incorporation; 1998.

21. Grupta SK, Pope GS. Variation in the level of progesterone in the systemic plasma of the cow. J Endocrinol 1968; 40:xii.

22. Mondal S, Prakash BS, Palta P. Peripheral plasma FSH concentration in relation to expression of estrus in Sahiwal cattle (Bos indicus). Indian J Physiol Pharmacol 2004; 48 (2): 245-250.

23. Solano R, Fernández O, Martínez G. Comportamiento del ciclo estral en novillas Holstein bajo condiciones climáticas de Cuba. Revista Cubana de Ciencias Veterinarias 1988; 19(1):47-59.

24. Hernández LJ, Padilla RF, Koppel RE, Roman $\mathrm{PH}$, Pérez SJ , Castillo RH. Comportamiento reproductivo del ganado bovino lechero en clima tropical. 6. Pérfiles de progesterona, estradiol y hormona luteinizante durante el ciclo estral en tres genotipos en dos estaciones del año. Téc Pecu Méx 1984; 47: 102-115.

25. Báez GM, Grajales H, Pérez JE. Caracterización del ciclo estral mediante perfiles de esteroides (progesterona, 17 $\beta$-estradiol) en la raza Costeño con Cuernos (Bos taurus) en el trópico Colombiano. Livestock Research for Rural Development [en línea] 2007; 19(9). [citado 3 Dic 2008]. URL Disponible en: http://www. Irrd.org/ Irrd19/9/baez19132.htm.

26. Grajales H, Hernández A, Prieto E. Edad y peso a la pubertad y su relación con la eficiencia reproductiva de grupos raciales bovinos en el trópico colombiano. Livestock Research for Rural Development [en línea] 2006 18(10) [citado 3 Dic 2008]. URL Disponible en: http://www.Irrd.org/Irrd18/ 10/graj18139.htm.

27. Mondal S, Prakash BS. Peripheral plasma progesterone concentration in relation to estrus expression in Sahiwal cows. Indian J Physiol Pharmacol 2003; 47(1): 111-114. 
28. Lamothe-Zavaleta C, Fredriksson G, Kindahl $\mathrm{H}$. Reproductive performance of Zebu cattle in Mexico. 2. Seasonal influence on the levels of progesterone, estradiol 17- $\beta$, cortisol and LH during the estrous cycle. Theriogenology 1991; 36(6):897-911.
29. Mai HM, Ogwu D, Eduvie LO, Voh Jr AA. Detection of Oestrus in Bunaji cows under field conditions. Trop Anim Health Prod 2002; 34:35-47. 